Ocularbeobachtung nachstehen wird. Sie gewinnt nur dadurch an Bedeutung, dass sie in den schwer sichtbaren Theilen der Spectra - Violett und Ultra - Violett - uns zur Kenntniss von stärkeren Absorptionsstreifen verhelfen kann. Uebrigens würde auch aus der vollendetsten Photographie, in Bezug auf die Breite der SpectralLinien, nur eine relative Messung der Linien untereinander möglich werden, aus welcher man keine Schlüsse auf die Rotation ziehen künnte, da die Breite der Linien auf verschiedenon photographischen Platten von der Länge der Exposition, der Empfindlichkeit des Processes und der Entwickelung der Platte abhängt.

Ich werde zum Schluss, zur möglichst vollständigen Erschöpfung des Gegenstandes, noch ausrechnen, welche Umdrehungsgeschwindigkeiten manche Sterne haben würden, wenn dic Linieu, welche uns in ihren Spectren verbreitert und verwaschen erscheinen, in Folge der Rotation verbreitert worden wären, und endlich, welche Intensitätsverhältnisse in den verbreiterten Linien stattfinden miissten.

Ich wälıle dazu die Sterne der 1. Klasse bei denen, wie schon oben bemerkt, die Wasserstofflinien stark verbreitert sind. Nach meinen Messungen*) ist die Breite der $F$-Linie, in Wellenlängen ausgedrückt, im Spectrum von a Lyrae 1.075 Mill. Millm. es würde diese Breite eine Geschwindigkeit eines Aequatorpunktes des Himmelskürpers von 45 geogr. Meilen voraussetzen. Ein Aequatorpunkt von a Aquilae, bei dem die Breite der F-Linie in Spectrum nur 0.600 Mill. Millm, beträgt, wïrde die immer noch ansehnliche Geschwindigkeit von $2 \overline{5}$ geogr. Meilen haben. Es erscheinen diese Geschwindigkeiten, zumal im Vergleich mit der Rotationsgeschwindigkeit unserer Sonne (Aequatorpunkt 0.27 geogr. Meilen) als in hohen Grade unwahrscheinlich. --

Unter der Voraussetzung des günstigsten Falls, dass die Rotations-Axe des Sterns senkrecht auf dem Visions- radius steht, werden Punkte der rotirenden Oberfläche um so mehr aut Verschiebung der Spectral-Linien wirken, je näher sie dem Aequator und der durch den Mittelpunkt des Sterns gehenden, senkrecht auf dem Visionsradius stehenden Ebene gelegen sind. In Folge dessen werden die linien verbreitert und an den Rändern verwaschen erseheinen müssen. Die Curve, welche die Intensitäten in verschiedenen Entfernungen von der Mitte der verbreiterten Spectral - Linie ausdrückt, ist durch folgende Zahlen gegeben:

\begin{tabular}{|c|c|c|c|c|c|}
\hline Entfernung & von der & Mitte & & & Intensität \\
\hline & \pm 0. & 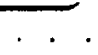 & . & & 1.00 \\
\hline & \pm 1 & & . & & 0.99 \\
\hline & \pm 2 & . & . & & 0.96 \\
\hline & \pm 3 & . & & & 0.91 \\
\hline & +4 & . & . & & 0.84 \\
\hline & $+\dot{5}$ & . & & & 0.75 \\
\hline & +6 & . & . & & 0.64 \\
\hline & +7 & . & & & 0.51 \\
\hline & $\pm s$ & & & & 0.36 \\
\hline & \pm 9 & . & & & .0 .19 \\
\hline & $\overline{+10}$. & . . & & & 0.00 \\
\hline
\end{tabular}

Die Curve fällt zuerst langsam dann aber sehr schnell ab, es würden die verbreiterten, an den Rändern mit einem sehr zarten allmälig verlautenden Halbschatten umgebenen Linien daher möglichst wenig geeignet sein, $z u$ einer, nur einigermassen genauen, Bestimmung der Rotation zu dienen; dazu kommt noch, dass die Intensität der verbreiterten Linien in der Mitte mit der $\mathrm{Zu}$ nahme der Rotationsgeschwindigkeit abnimmt. Wenn die Linie bei der Rotationsgescliwindigkeit 0 , die Intensitat 1 hat, so wird bei einer Geschwindigkeit, welche die Linie um das Doppelte ihrer Ausdehnung verbreitert, die Intensität in der Mitte nur $3 / 4$, bei vierfacher Breite $2 / 7$ und bei sechsfacher Breite nur $1 / 6$ betragen.

Astrophys-Observatorium zu Potsdam Juni 1877.

s) Astr. Nachr. No. 1963 .

\title{
Stars to be observed with Mars at Opposition 1877.
}

To determine the diurnal parallax of various planets the following Comparison stars will be observed at the Island of Ascension in connection with the Expedition of the Royal Astronomical Society. I propose to make a Heliocentric triangulation of the Comparison Stars and to combine this with meridian-observations. I hope in this way to obtain relative places so exact that the errors of the Star places may be neglected in the final discussion for parallax. The Subscriber earnestly requests the cooperation of Observatories possessing good Meridian-Instruments. Extra-MeridianObservations of difference of $A R$ and Decl, will also be of very great value. 


\begin{tabular}{|c|c|c|c|c|c|c|c|c|c|c|}
\hline \multirow[t]{2}{*}{$\begin{array}{l}\text { Adopted } \\
\text { Name } \\
\text { of Star. }\end{array}$} & \multicolumn{2}{|c|}{$\begin{array}{l}\text { Approximate } \\
1877.0\end{array}$} & \multirow[t]{2}{*}{$\begin{array}{l}\text { No. in } \\
\text { Weisse's } \\
\text { Bessel. }\end{array}$} & \multirow[b]{2}{*}{ Mag. } & \multirow{2}{*}{$\begin{array}{l}\text { Mag. in } \\
\text { Berlin Academy } \\
\text { Star. Cat. }\end{array}$} & \multirow[t]{2}{*}{$\begin{array}{l}\text { No. in } \\
\text { Lalande. }\end{array}$} & \multicolumn{3}{|c|}{$\begin{array}{l}\text { No. in } \\
\text { Santini. }\end{array}$} & \multirow[t]{2}{*}{ Remarks. } \\
\hline & & & & & & & Mag. & & Mag. & \\
\hline $\mathbf{a}$ & $22^{\mathrm{h}} 47^{\mathrm{m}} \mathrm{O}^{\mathrm{s}}$ & $-12^{\circ} 16^{\prime}$ & XXII. 956 & 7 & 6 & 44756 & 6 & 2577 (1858) & 7 & \\
\hline $\mathbf{b}$ & 22.47 .38 & -12.50 & 966 & $7-8$ & $7-8$ & $\ldots$ & 8 & $2140(1862)$ & $7-8$ & \\
\hline$\alpha$ & 22.51 .37 & -11.47. & 1047 & 9 & & & & & & \\
\hline c & 22.53 .7$. & -13.44 & 1079 & 6 & 7 & 44937 & $6 \frac{1}{\square}$ & 2145 (1862) & $6-7$ & \\
\hline$\beta$ & 22.56 .4 & -11.55 & 1156 & 8 & 8 & 45050 & 8 & $2591(1858)$ & $8-9$ & Lal. has Dec. $-10^{0} 55^{\prime}$ \\
\hline$\mu$ & 2258.3. & -12.50 & 1204 & 8 & 8 & $\ldots$ & $\ldots$ & $2154(1862)$ & 8 & $\begin{array}{l}\text { Schj. } 22^{\mathrm{b}} 58^{\mathrm{m}} 2^{\mathrm{s}} 91 \\
-12^{0} 50^{\prime} 26^{\prime \prime} 8(8-7)\end{array}$ \\
\hline$d$ & 22.59 .28 & -11.6 & 1232 & 8 & $7-8$ & 45169 & 7 & 25094 (1858) & 8 & \\
\hline$y$ & 23. 0.30 & -13.23 & 1249 & 8 & $8-9$ & 45197 & 9 & & & \\
\hline e & 23. 0.58 & -12.28 & 1261 & 8 & 8 & 45213 & 8 & 2599 (1858) & $7-8$ & \\
\hline 8 & $23 . \overline{\text { ร. } 3 .}$ & -11.10 & XXIII. 49 & 9 & & & & & & \\
\hline$\varepsilon$ & 23.5 .32 & -12.36 & 57 & 8 & 8 & 45380 & 7 & & & \\
\hline$f$ & 23.8 .16 & -11.21 & 123 & 7 & 7 & 45490 & 6 & & & \\
\hline $\mathbf{g}$ & 23. 8.55 . & -12.14 & .. & $\ldots$ & $7-8$ & 45504 & $7 \frac{1}{2}$ & & & \\
\hline$\psi^{\prime} \stackrel{\circ}{\mathrm{Aq}}$ & .23 .9 .27$. & -9.45 . & $\ldots$ & $4-5$ & & & & & & \\
\hline $\mathrm{h}$ & 23.11 .15 & -12.23 & XXIII.185 & 7 & 7 & 45582 & 6 & $2618(1858)$ & 7 & \\
\hline $\mathbf{i}$ & 23.12 .34 & -10.17 & 226 & $\overline{\mathrm{j}}-6$ & 5 & 45628 & 6 & $2622(1858)$ & $5-6$ & $\psi^{3}$ Aquarii. \\
\hline $\mathbf{k}$ & 23.12.37. & -12.51. & XXIII.228 & 8 & 8 & 45633 & 7 & $2177(1862)$ & 7 & \\
\hline 1 & 23.14 .29 & -11.12. & 265 & 8 & 8 & 45708 & 8 & $2625(1858)$ & 8 & \\
\hline$\lambda$ & 2316.36 . & -11.27 & 309 & 8 & $8-9$ & 45777 & 8 & $2627(1858)$ & 8 & \\
\hline$\zeta$ & 23.16 .55 & -10.4 & 315 & 8 & 8 & 45789 & $\gamma$ & . & $\ldots s$ & $\begin{array}{l}\text { Schj. } 23^{\mathrm{h}} 16^{\mathrm{m}} 54^{\mathrm{s}} .26 \\
-10^{0} 3^{\prime} 33^{\prime \prime} .1(8-7)\end{array}$ \\
\hline$\eta$ & 23.20 .14 & -10.43. & 377 & 8 & & & & & & \\
\hline $\mathbf{m}$ & 23.21 .40 & -12.7. & 402 & 7 & 7 & 45937 & 6 & 2634 (1858) & 7 & \\
\hline $\mathrm{n}$ & 23.22 .39 & -9.57 . & 427 & 7 & 7 & 45965 & $6 \frac{1}{2}$ & $2636(1853)$ & 7 & \\
\hline$q$ & 23.26 .4 & -11.41. & 497 & 7 & 7 & 46090 & $6 \frac{1}{2}$ & 2643 (1858) & 7 & \\
\hline $\mathbf{r}$ & 23.29 .6 . & -11.14 & 571 & 8 & 8 & $\ldots$ & $\ldots$ & $2653(1858)$ & 8 & \\
\hline $\mathbf{s}$ & 23.29 .40 & -9.27 & 586 & $7-8$ & $7-8$ & 46229 & $7 \frac{1}{2}$ & $\ldots$ & & Schj. $23^{\mathrm{h}} 29^{\mathrm{m}} 39^{\mathrm{s}} .37$ \\
\hline $\mathbf{t}$ & 23.31 .52 . & -9.19 & 629 & 7 & 7 & 46296 & $7 \frac{1}{2}$ & & & \\
\hline
\end{tabular}

Stars to be observed with Ariadne at Opposition 1877.

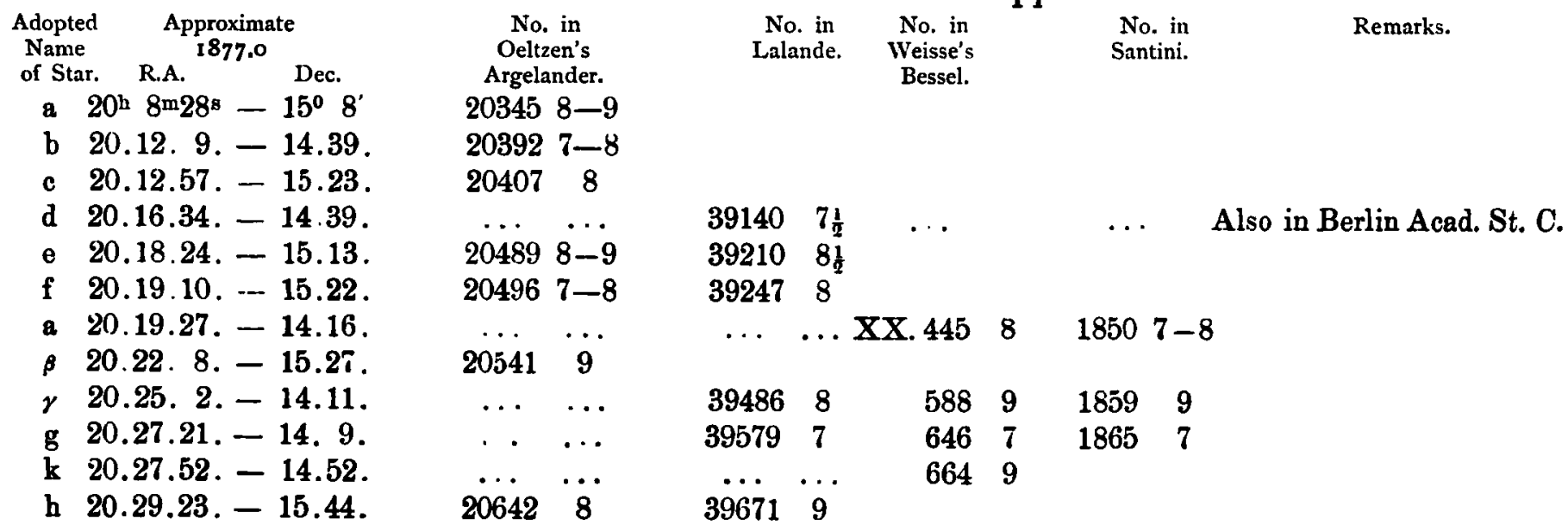




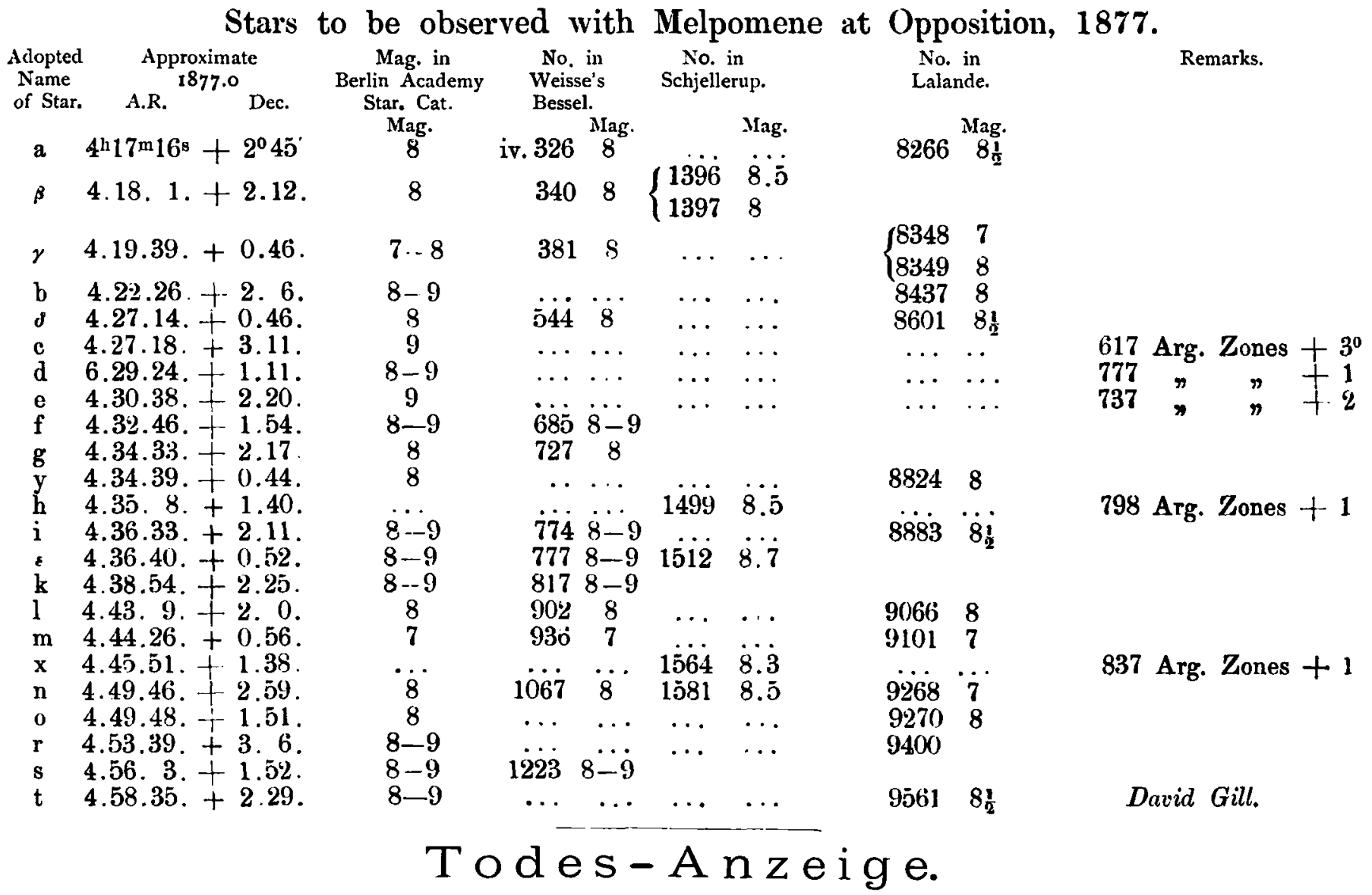

\section{Giovanni Santini.}

Ad un' ora pomeridiana del 26 corr. morì nella sua villa presso Padova il Prof. Giovanni Santini, nella grave età di oltre 90 anni. Nato a Caprese nella Provincia di Arezzo, il 30 gennaio 1787, Studiò Astronomia a Pisa con Piazzini e a Milano sotto la direzione di Oriani e Cesaris. In novembre del 1806 fu nominato Astronomo Aggiunto e nel 1813 prof. ordinario di Astronomia e Direttore di quest' Osservatorio. Nel $1853 \mathrm{fu}$, dietro sua domanda, dispensato dall' obligo di dare pubbliche lezioni, ma dopo la morte del Trettenero, avvenuta nel 1863, Egli le riprese spontaneamente e continuò a darle per altri due anni e mezzo.

Non conviene qui, nè sarebbe possibile in brevi linee, dare una notizia dettagliata sull' attività scientifica del Santini: per formarsene una idea basta pensare, che Egli fu uno dei più assidui osservatori e calcolatori, e che la sua vita scientifica abbraccia il periodo di tempo compreso fra il 1807 e il 1870. A Lui sono dovate numerosissime osservazioni di comete, di pianeti, di occultazioni, di eclissi, molti calcoli di orbite, alcuni cataloghi stellari, una quantita di pregevolissime memorie sparse negli atti di insigni Accademie e due opere magistrali: gli Elementidi Astronomia e la Teorica degli Stromenti Ottici. I lettori delle Astronomische Nachrichten hanno un saggio della sua attivita nelle numerose comunicazioni da Lui inviate a questo giornale e contenute nei primi 60 volumi dello stesso.

Oltre che per i suoi meriti scientifici, il Santini deve essere ricordato pei servigi da Lui resi alla istrazione della gioventù come insegnante e come Direttore della Facoltà Matematica di questa Università, per la eccellenza del suo carattere e per le sue virtù civili e domestiche, che lo resero universalmente amato e rispettato.

Nell' Osservatorio di Padova, che a Lui deve il suo maggior lustro e che conserva tanti documenti della sua instancabile operositè, il Santini lascia una memoria cara e venerata.

Dall' Osservatorio Astronomico di Padova, il 28 giugno 1877 .

G. Lorenzoni, Astronomo Aggiunto. 